\title{
Filigrane
}

Écoutes psychothérapiques

\section{Les capteurs de rêves}

\section{Daniel Puskas}

Volume 17, numéro 2, automne 2008

L’avenir du clinicien II

URI : https://id.erudit.org/iderudit/019422ar

DOI : https://doi.org/10.7202/019422ar

Aller au sommaire du numéro

Éditeur(s)

Revue Santé mentale au Québec

ISSN

1192-1412 (imprimé)

1911-4656 (numérique)

Découvrir la revue

Citer cet article

Puskas, D. (2008). Les capteurs de rêves. Filigrane, 17(2), 110-121.

https://doi.org/10.7202/019422ar

\section{Résumé de l'article}

L'auteur témoigne de son parcours comme psychoéducateur auprès de jeunes en difficulté, métier qu'il exerce en parallèle à celui de psychanalyste, et qui lui a permis de se familiariser avec des modes de fonctionnements psychiques particuliers. Ses deux métiers se nourrissent l'un de l'autre.
Ce document est protégé par la loi sur le droit d'auteur. L’utilisation des services d’Érudit (y compris la reproduction) est assujettie à sa politique d'utilisation que vous pouvez consulter en ligne.

https://apropos.erudit.org/fr/usagers/politique-dutilisation/
Cet article est diffusé et préservé par Érudit.

Érudit est un consortium interuniversitaire sans but lucratif composé de l’Université de Montréal, l'Université Laval et l'Université du Québec à Montréal. Il a pour mission la promotion et la valorisation de la recherche. https://www.erudit.org/fr/ 


\title{
Les capteurs de rêves
}

\author{
daniel puskas
}

\begin{abstract}
L'auteur témoigne de son parcours comme psychoéducateur auprès de jeunes en difficulté, métier qu'il exerce en parallèle à celui de psychanalyste, et qui lui a permis de se familiariser avec des modes de fonctionnements psychiques particuliers. Ses deux métiers se nourrissent l'un de l'autre.
\end{abstract}

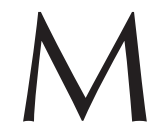

on fils, âgé peut-être de deux ans, alors que nous nous arrêtions dans une station-service, me dit: «On va faire le plein du sens ». Bien sûr, il signifiait le plein d'essence. Ce joli mot d'enfant est demeuré dans la famille. Et lorsque j'y repense aujourd'hui, moi aussi, petit, je voulais faire le plein du sens. Je partis ainsi, dans l'existence, avec mon baluchon au sein duquel j'avais enfoui, comme des objets précieux, certaines questions. J'en déballai quelques-unes lors de ma psychanalyse. Un certain nombre, mystérieuses, surgissent encore parfois aux détours de ma vie. J'essaie de créer, de leur façonner un sens.

La théorie et l'expérience psychanalytiques me sont toujours apparues comme susceptibles de fournir des réponses à plusieurs de mes interrogations. C'est pourquoi, d'aussi loin que je me souvienne, j'ai voulu devenir psychanalyste.

Mais, plusieurs l'ont déjà fait remarquer, nous devons exercer une autre profession avant celle de psychanalyste. Lorsque vint le temps de choisir un métier, je retins celui de psychoéducateur. Cette profession conjugue une double centration, psychologique et éducative. Dans ce confluent se joignent deux passions miennes. En effet, j'accorde un intérêt marqué pour la vie psychique et pour l'enseignement, la transmission.

La formation en psychoéducation et sa pratique s'avèrent d'une grande richesse. Mes premières années comme psychoéducateur se déroulèrent en centres de réadaptation, comme on les nommait à l'époque, nommément au Centre d'Orientation. Cet établissement traitait des garçons de la période de latence présentant de graves problèmes d'adaptation et possédant un potentiel intellectuel supérieur à la moyenne. Ces années furent très formatrices. J'ai pu observer certaines pathologies au moment même de leur structuration. J'ai pu entendre la terrible souffrance d'enfants abandonnés et comprendre comment ils s'en protégeaient par l'érection d'efficaces mécanismes de défense contre l'angoisse d'abandon et la dépression. J'ai vu également certains garçons décompenser et tenter de se reconstruire avec des délires. Tout cela au quotidien.

Je n'oublierai jamais certains moments. Une récréation se termine, je fais entrer les enfants. Il me manque André, un nouveau. Où est-il? Une fugue? 
Je le vois arriver, il marche lentement, sa tuque enfoncée, il regarde par terre.

Je lui demande: «André, que faisais-tu?»

Il relève sa tête et répond très calmement: «C'est 3 à 2 pour l'ouest. »

Il remarque sans doute ma perplexité et il me donne les explications de son absence. André était un garçon singulier, très isolé. Il connaissait par cœur l'ensemble du réseau de transport de l'île de Montréal. Aux nouveaux stagiaires, il lançait des défis: «Donne-moi une adresse et je vais te dire le trajet des autobus et métros.» Il ne se trompait jamais, même devant les colles les plus difficiles. Comme il n'aimait pas beaucoup la fréquentation des autres garçons, durant cette récréation il avait été regarder les autobus sur le boulevard Gouin. Il compta celles qui se dirigèrent vers l'est, et vers l'ouest. Cette fois-là, l'ouest gagna 3 à 2 .

André vivait dans un univers bien à lui. Intuitivement, je sentais le besoin qu'il éprouvait de bien connaître, l'ensemble du système de transport. Il manquait de points de repère, les espaces et les temps qu'il ne pouvait prévoir, donc maîtriser, l'angoissaient au plus haut point. Il avait besoin de ritualiser son quotidien.

Je développai avec André une relation significative. Je savais aménager l'environnement autour de lui pour organiser son monde intérieur. À un moment, je dus m'absenter quelques semaines, pour une raison de santé. À mon retour, l'équipe me dressa un portrait plutôt sombre : il avait beaucoup régressé. Nous reprîmes nos rencontres hebdomadaires. Lors de la première, il sut en quelques mots me faire comprendre l'état de détresse dans lequel il avait vécu: «quand j'ai su que tu revenais j'ai rêvé, m'a-t-il dit d'un ton posé. J'étais dans le brouillard, je conduisais une auto, je ne voyais rien. C'était blanc et gris partout. J'avais peur de mourir dans un accident. À un moment donné, mes essuie-glaces ont démarré, c'était comme si ça enlevait de la neige, là je t'ai vu, tu me faisais des signes pour m'indiquer où aller, ça m'a rassuré tout de suite, il n'y avait plus de danger. »

La clinique du psychoéducateur est un travail avec la psychopathologie de la vie quotidienne. Nous observons dans la vie de tous les jours des manifestations morbides et intervenons dans le réel, l'imaginaire et le symbolique.

Par exemple, en étude de cas nous avions vu que Joseph ressentait des angoisses de morcellement importantes. J'avais bien compris intellectuellement. Mais c'est en vivant un moment de routine avec lui que j'allais vivre une expérience où comprendre n'est plus seulement une question cérébrale.

Je travaillais en soirée, j'animais le moment des douches. Au tour de Joseph, après quelques minutes il se met à hurler, jamais je n'oublierai ce cri. Je me précipite afin de fermer la douche, Joseph s'arrête sur-le-champ. Ce petit garçon de huit ans est nu devant moi, livide. Je l'enserre avec une large serviette. Le frotte.

«Tu as eu peur?» lui dis-je.

Il me regarde, hoche de la tête et dit: «Oui, je me suis savonné, j'ai fait le bonhomme de neige comme Josée nous l'a montré, plein de savon partout. Lorsque j'ai vu que la mousse du savon passait dans le trou de la douche, j'ai eu peur que toute ma peau se détache, pis mes os et ma tête. » Joseph avait eu peur de fondre dans la douche, j'avais entendu le cri d'un enfant qui se sent disparaître, qui 
se morcelle. Je l'enserre de nouveau dans sa serviette, comme on emmaillote un bébé dans ses langes, et le masse afin qu'il sente son corps, la vie qui bat en lui, son unité retrouvée. Il s'apaise lentement. Je ne dis pas un mot.

Ce travail de psychoéducateur me passionnait, mais je voulais aussi vivre l'expérience psychanalytique. Lorsque je reçus ma première paye, j'ai contacté un psychanalyste didacticien, dans le but d'entreprendre une analyse personnelle. J'engageai cette aventure exceptionnelle avec $\mathrm{D}^{\mathrm{r}}$ Julien Bigras.

Avant de démarrer ma pratique privée, je continuai à travailler dans les centres de réadaptation. Pendant une dizaine d'années, j'œuvrai auprès de familles qui adressaient leur demande à ces centres. J'y ai approfondi l'approche systémique, supervisé par des professionnels de l'Hôpital Juif de Montréal qui avaient développé une expertise en thérapie familiale. C'est là, dans le travail auprès de familles, que j'ai pu observer les effets pathogènes des messages paradoxaux, de conflits de loyauté ou encore des injonctions parentales délétères.

Ainsi, Luc, un adolescent, présente de graves difficultés comportementales. Il commence à fréquenter, ce que les intervenants de l'école soupçonnent être des gangs de rue. Il consomme et vend des drogues douces. Luc n'est pas placé dans un Centre, c'est le praticien social qui nous le réfère. Nous convenons de convoquer la famille. Luc est enfant unique. Sa mère, une personne effacée, travaille comme commis dans une PME. Son père possède une forte personnalité avec des traits narcissiques importants; il est vendeur. Le couple se dit sans histoire, mais il est clair, après quelques minutes seulement, que Monsieur domine sa femme qui ne prend aucune initiative sans le regarder. Luc est assis entre les deux. Lorsque nous leur demandons quel est le problème, monsieur nous donnera la même version que l'information que nous avions reçue de l'école et du travailleur social. Luc a de mauvaises fréquentations et cela l'inquiète, il est peut-être pris dans un engrenage. Malgré son bagout, Monsieur ne nous convainc pas. Placé devant certaines incongruités, il finira par avouer que le problème, ce n'est pas tant son fils que les coups de téléphone répétés de l'école. Ces appels le dérangent profondément. Madame demeure vague, affirmant son amour pour son fils. En fait, elle pardonne tout à son fils, blâmant l'école pour son manque d'encadrement. Une seconde rencontre est planifiée pour la semaine suivante. Une demi-heure avant son début, la mère nous téléphone. Monsieur ne pourra se présenter à la séance, il fait beau et il a reçu une invitation pour aller jouer au golf avec un collègue, une belle occasion d'affaires. Elle nous dira que son fils lui a promis de faire des efforts et que tout devrait rentrer dans l'ordre dans les semaines qui suivront. La famille ne formule donc plus de demande d'aide.

Nous utilisons le temps de la séance afin de discuter du cas en équipe. Notre façon de travailler consiste à formuler des hypothèses. Nous ouvrons des alternatives, nous ne voulons pas travailler avec des certitudes. C'est pourquoi nous aimons mettre sur la table plusieurs hypothèses selon la formule du remueméninges. Par exemple, le message transmis par les parents à leur fils, est qu'il peut faire n'importe quoi, pourvu qu'il ne les dérange pas. Une autre possibilité: 
le fils agirait certains désirs du père. Il y aurait un accord tacite entre eux, comme si le père disait à son fils: «Sois un bon vendeur comme moi et tu t'en sortiras toujours.» Nous n'écartons pas plus la possibilité d'une complicité inconsciente avec la mère, le fils pourrait agir ce qu'elle ne se permet pas elle-même. Sans parler des enjeux oedipiens, elle l'amènerait à être un fils à maman ou vivre dans un système familial où la loi du père est pervertie, etc.

Des années effervescentes: le modèle psychoéducatif, l'approfondissement de la théorie psychanalytique, ma psychanalyse personnelle et la pratique systémique. Des années où la seule limite à ma curiosité de cliniciens était le temps. Au Centre Jeunesse, comme on le nomme maintenant, je suis devenu un psychoéducateur avec une compréhension psychanalytique des dynamiques individuelles, une écoute analytique, travaillant aussi sur le système et la dynamique familiale. Ma formation en psychoéducation m'a particulièrement bien préparé à travailler avec des sujets très hypothéqués sur le plan narcissique, en axant une partie de mes interventions sur les forces et les mécanismes de résilience.

C'est donc en m'appuyant sur ce triptyque conceptuel - psychoéducatif, psychanalytique et systémique - que je réfléchis à mon travail clinique en Centre Jeunesse et tente de le conceptualiser. Je travaille maintenant depuis plus de trente années en réadaptation, dont les quinze dernières comme consultant clinique auprès des équipes éducatives. Avec celles-ci, mes défis se situent à deux niveaux : d'abord celui de cerner la nature des difficultés en formulant des hypothèses de travail, puis celui de traduire dans la quotidienneté un plan d'intervention thérapeutique pour ces jeunes et leurs familles.

Plus je tente de faire un bilan de mes années de travail, plus j'en arrive à me définir d'abord comme un clinicien. Je suis un professionnel avec des formations complémentaires. Devant un problème clinique, je réfléchis avec l'ensemble de mon bagage conceptuel et de mon expérience. Je ne peux pas me définir en fonction du lieu où j'exerce. À mon cabinet, je ne suis pas seulement psychanalyste, au Centre jeunesse je ne réfléchis pas seulement comme un psychoéducateur.

D'autant que les jeunes placés dans des Centres jeunesse sont des sujets avec des histoires de vie très complexes, empreintes d'abus et de négligence, et vivant dans des systèmes familiaux très pathogènes. Sur le plan psychopathologique, des diagnostics psychiatriques accompagnent de plus en plus souvent les grandes difficultés d'adaptation psychosociales et comportementales (désordres de la conduite) de ces jeunes.

Depuis des années, je travaille des problématiques qui me sont apparues importantes sur le plan clinique. J'examinerai ici celles de la fonction paternelle, de la répétition par la mise en acte, centrale dans le transgénérationnel et la rencontre d'accompagnement clinique.

\section{La fonction paternelle}

Cette problématique se déploie de façons diverses dans le travail clinique en réadaptation. J'ai pu constater dès le début de ma pratique que la fonction pater- 
nelle défaillait souvent dans ces familles. J'ai observé qu'une majorité de mères avaient du mal à faire une place à un tiers et, même si souvent les pères étaient présents, ils n'incarnaient pas la fonction symbolique d'un père, ils étaient souvent agresseurs ou négligents. Comment alors, comme intervenant, s'installer dans cette fonction paternelle ? Occuper cette place de tiers sans susciter la rivalité avec la mère ? Comment intervenir dans les familles à transactions perverses, sans être complice? Mes observations cliniques me confrontaient à des questions théoriques qu'il me fallait tenter de comprendre.

En fait, le père titularise trois fonctions: celles de géniteur, de nourricieréducateur et de donneur de nom, garant des règles d'alliance et de filiation. C'est principalement cette dernière fonction qui m'intéresse, à savoir en d'autres termes: la loi de l'interdit de l'inceste. Comment installer cette loi dans les familles du Centre ? Car l'important ne consiste pas tant de rappeler cette loi à ces enfants et à ces parents qu'à produire, pour reprendre les mots de Françoise Dolto, une castration symboligène. En effet, rien ne sert de sortir les «gros bras», de rappeler les lois de nos sociétés en tapant sur les personnes. Bien sûr, il nous faut comme représentant de ces lois nommer les interdits, dans le respect de l'autre et dans un accompagnement qui amènera, dans le meilleur des cas, à symboliser cette loi. L'important ici n'est pas de changer les comportements, car à l'intérieur d'une famille, les échanges, les relations, sont chargés de désir. Et ces désirs de l'autre demandent à être régulés, c'est-à-dire soumis à une règle, à une loi. Cette loi est défaillante ou pervertie dans les systèmes familiaux auprès desquels nous travaillons et l'enfant ne possède souvent pas de point de repères pour se construire. Donc, on le voit, le défi posé est de taille: il s'agit de toucher les dynamiques individuelles et interactionnelles. Notre travail porte sur les désirs, pas seulement sur les besoins et les comportements.

En fait, l'ensemble d'un processus structurant pour l'enfant pose problème dans ces familles. Il s'agit donc aussi d'installer un cadre. Ma formation en psychoéducation m'a été d'une formidable utilité dans l'organisation du cadre des séances en thérapie familiale, du cadre de vie des familles dans leur quotidien et du cadre de vie en internat de réadaptation. Cette organisation consiste en une série d'actions professionnelles à réaliser sous forme de séquences nécessaires à une intervention de réadaptation: observer, évaluer, planifier, animer et utiliser. Un cadre dans lequel je dois comprendre les interactions et leurs effets sur la vie psychique consciente et inconsciente.

Donc, dans mes interventions au Centre jeunesse, j' ai eu à me poser la question de comment incarner une fonction paternelle dans ces familles. J'en suis rapidement parvenu à identifier que le problème observé dans le présent s'inscrivait dans une histoire. Ces parents avaient eu des parents et la carence de la fonction paternelle se structurait dans une genèse. Avec ce constat, je me mis à penser l'histoire individuelle sur au moins trois générations. Et c'est lorsque j'ai été confronté au travail auprès d'une clientèle d'adolescentes placées en centre de réadaptation, en vertu de la loi de la protection de la jeunesse, que j' ai pu observer, avec les équipes 
éducatives avec lesquelles je travaillais, une répétition par les filles des problèmes vécus par leurs mères. J'en suis arrivé à postuler l'existence d'une compulsion de répétition transgénérationnelle ${ }^{1}$ par défaut de la fonction paternelle.

Dans mon livre Amours clouées (2002, 23), j'articule la fonction paternelle avec la problématique de la répétition de la façon suivante:

«Lorsqu'on se met à étudier la question de la répétition transgénérationnelle, on constate rapidement qu'elle est partout présente dans le fait humain, c'est même l'une des pierres d'assise qui le constitue. Le transgénérationnel est intimement lié à l'histoire et de ce fait est d'une valeur structurante indispensable à la construction de l'identité chez l'homme. En effet, qu'est-ce qui traverse les générations? Ce sont les structures sociales, les lois, le langage, les arts, les patronymes. C'est tout ce qui fait que l'homme se constitue homme. Le complexe d'Edipe, par son universalité, est, en ce sens, exemplaire. Il est le creuset par lequel se construit l'identité d'une personne et le processus par excellence de la transmission entre les générations. Le conflit œdipien est l'espace de transmission des interdits, des valeurs, des idéaux, des identifications, et l'articulation de la fonction paternelle en constitue la pierre angulaire.»

Il existe donc une clinique du transgénérationnel dont j'ai posé les jalons dans cet ouvrage. J'y traite des mécanismes qui opèrent dans la répétition intergénérationnelle. Dans un premier temps, je décris les mécanismes intrapsychiques. Par exemple, dans les situations traumatiques, telles les incestes, des mécanismes inconscients œuvrent: déni, refoulement, dissociation massive. Ces mécanismes sont, avec la mise en acte des traumas, à la base de la compulsion de répétition transgénérationnelle. De plus, dans les troubles de l'attachement, les patterns relationnels intériorisés chez les personnes souffrantes sont répétés par la génération suivante. Les mères en trouble d'attachement développent avec leur bébé des liens anxieux ou d'évitement et répètent le type de liens qu'elles ont elles-mêmes vécu.

Enfin, j' ajouterai que l'importance de la reconstruction d'une histoire de vie sur trois générations m'est apparue essentielle à ma clinique et l'utilisation du génogramme, un outil indispensable pour la penser. Ce dernier constitue une façon de dresser l'arbre généalogique d'une famille. Il contient des informations sur les membres de cette famille et leurs relations réparties sur au moins trois générations. Ces diagrammes familiaux dressent une carte des relations et des modèles de fonctionnement et fournissent des informations graphiques de la famille. Ils permettent d'obtenir une image de modèles familiaux complexes. Individu, identité, fonction et contexte deviennent les points essentiels de l'analyse du génogramme. Une lecture systémique en permet une bonne compréhension (McGoldwich, M., Gerson, R.,1990). L'utilisation de cette technique permet de tracer les frontières 
physiques et émotives d'une famille, de décrire les caractéristiques de ses membres et de repérer la petite histoire des événements importants. Le génogramme dépeint et recense ainsi la qualité et la quantité des options relationnelles vécues entre les membres d'une famille (Guerin, Pendagast, 1976).

Un tel parti pris permet de contextualiser et, comme le souligne Morin ${ }^{2}$, de reconnaître en même temps le singulier, l'individuel. Contextualiser veut dire tenir compte du et des contextes: social, national, historique, migratoire, familial, de la petite comme de la grande histoire. En tenir compte, soit, mais pas comme données objectives, plutôt comme réalité construite. Ajoutons que cette construction n'est jamais bâtie une fois pour toutes, elle s'élabore continuellement, et souvent dans l'après-coup des événements. Ainsi en est-il par exemple du moment où je suis devenu père. Beaucoup de souvenirs me sont revenus à propos de mon père et moi et je les revoyais avec un autre œil. Je pourrais ajouter qu'il en a été de même à chaque âge de mes enfants; $\mathrm{j}$ 'ai réorganisé mon histoire toujours en fonction de la nouvelle place que j'occupais, à savoir la place de père et non plus seulement de fils.

Le hasard a aussi fait que ma formation en psychanalyse puisse également servir dans l'utilisation particulière que je fais du génogramme. En effet, l'approche du génogramme élaborée par Sylvana Montagano diffère, quant à sa finalité, du génogramme traditionnel. Cette auteure italienne présente une approche au confluent de plusieurs courants de pensée. Psychologue de formation, psychanalyste, thérapeute conjugale et familiale, elle donne une place centrale au sujet, à la personne demandant de l'aide.

Bref, le bilan de mon parcours dans l'étude de la problématique du transgénérationnel et de la question paternelle me permet de prendre conscience que, parallèlement à une élaboration conceptuelle, je suis préoccupé par le travail concret. C'est pourquoi l'outil du génogramme revêt une importance dans ce parcours. Il devient un outil pour penser. Pour m'être utiles, les concepts doivent posséder une vie concrète; c'est peut-être dû ma formation en psychoéducation qui me force à opérationaliser mes réflexions théoriques. C'est comme si je me disais : «Bon, maintenant qu'on le comprend mieux, comment pourrait-on intervenir concrètement (dans le symbolique l'imaginaire et le réel).»

\section{La répétition et la mise en acte}

Le travail clinique auprès de la clientèle des Centres jeunesse exige qu'une large place soit accordée au repérage des passages à l'acte dans l'histoire familiale. J'ai pu souvent observer qu'un trauma vécu par la mère était littéralement remis en acte par elle auprès de sa fille d'une façon tout à fait inconsciente. C'est pourquoi j'attache une grande importance au repérage et à la compréhension des agirs dans mon travail clinique.

En voici un exemple.

Dès sa première entrevue Virginie raconte: «Mais, maman, j'en sais pas mal plus que tu penses. Je sais que ce qui m'est arrivée, ça t'es arrivée. C'est mamie qui m'a racontée.» La mère me regarde, "Monsieur me dit-elle solennellement, 
aidez-nous. Je ne voulais pas que ma fille soit victime d'inceste comme je l'ai été. J'ai rompu avec son père parce que j'avais peur, j'avais peur quand il lui donnait son bain, quand il la prenait sur ses genoux, la nuit quand il se levait, j'avais peur tout le temps. Le climat est devenu invivable, il est parti. J'avais peur quand nous allions chez mes parents, peur de ce que mon père pourrait lui faire. J'ai appris après que ma mère savait. Moi j'avais honte. Je me disais que c'était de ma faute. Ma mère a été hospitalisée à quelques reprises, j'étais l'aînée, j'ai pris des responsabilités, j'arrêtais l'école pendant ce temps, popotes, ménages, lavage. Enceinte, je suis partie avec le père de Virginie. Virginie, secrètement Virginie, c'était vierge, ma fille sera vierge, pas un crisse d'homme était pour la toucher. Virginie, tout pouvait être recommencé à neuf, je me refaisais une virginité. Je n'ai pas voulu la croire lorsqu'elle m'a dit que Mario l'avait touchée, ça faisait trop mal, ni vu ni connu, comme on dit. C'était la dernière chose que je voulais qui arrive à ma Virginie. Il y a eu d'autres hommes, je suis une dépendante affective vous savez.»

Virginie regardait sa mère. Elle n'avait rien appris de ce que sa mère venait de dire. Elle avait écouté pour la première fois l'émotion de sa mère. Elle croyait que sa mère voulait la protéger, mais elle croyait aussi, paradoxalement, que sa mère n'avait pas voulu la croire. Malgré qu'elle aimait sa mère, Virginie sentait, en même temps, qu'elle avait raison de lui en vouloir. (Puskas, 2002, 5)

Cette histoire n'est pas unique. La situation de madame Sirona incarne malheureusement l'histoire de beaucoup de familles des Centres jeunesse. Les événements traumatiques seront donc agis au lieu d'être remémorés, c'est ce que désigne le concept de mise en acte. Laplanche et Pontalis le définissent dans leur Vocabulaire: «comme un fait par lequel le sujet, sous l'emprise de ses désirs et fantasmes inconscients, les vit dans le présent avec un sentiment d'actualité d'autant plus vif qu'il en méconnaît l'origine et le caractère répétitif.»

La personne met en scène ce qu'elle pense être une nouvelle pièce de théâtre, elle ignore qu'elle n'en est aucunement l'auteur, elle n'est qu'un acteur d'une pièce déjà écrite. Ainsi un parent victime d'abus, soit de traumatisme dans l'enfance, pourra bien inconsciemment mettre en acte cette situation d'abus lorsqu'il aura des enfants. Le parent mettra en acte afin de s'éviter la remémoration, et en même temps pour comprendre et maîtriser ce qu'il ne comprend pas de son traumatisme vécu.

Je pointe ici tout le délicat travail de liaison à effectuer avec ces sujets. Il s'agit de relier le dit, le senti et l'agi.

\section{Les capteurs de rêve}

Le troisième thème que je développerai, à propos de mon parcours clinique en Centre jeunesse, est celui de la rencontre individuelle entre un éducateur et un jeune qu'il a la responsabilité d'accompagner. Un livre, Les capteurs de rêve: la construction de l'espace thérapeutique dans les rencontres d'accompagnement clinique, traitant du travail en réadaptation dans les centres jeunesse paraîtra sous 
peu. Ce titre m'a été inspiré, par les intervenants ${ }^{3}$ que je côtoie dans ma pratique professionnelle et, comme amis, depuis plus de trente ans.

Le capteur de rêves, cet instrument du quotidien aux pouvoirs magiques ${ }^{4}$, placé au-dessus du lit du dormeur et servant à capter ses rêves, prend sa source dans l'imaginaire amérindien. Un proverbe des Premières Nations affirme que : «Tous ceux qui connaissent le succès ont d'abord rêvé à quelque chose. »

L'intervenant des Centres Jeunesse incarne, pour moi, un capteur de rêves. Cette métaphore révèle son aspect protecteur: la captation des cauchemars dans les fils qui les retiennent. C'est l'une des tâches essentielles dévolues par notre société, entre autres, aux éducateurs. En plus, le capteur de rêves laisse passer, en son centre, les bons rêves. Il s'agit ici des activités oniriques bien sûr, mais surtout de l'espoir à capter, à porter, pour chacun de ces jeunes et leurs familles.

Enfin, le capteur conçu pour attraper les rayons du soleil, peut aussi être interprété comme une révélation du monde invisible. Les éducateurs interprètent et révèlent, en l'éclairant, un autre univers à des enfants, à des jeunes et leur famille qui souvent ne connurent qu'un seul monde, qu'une seule et bien triste réalité, sans magie, sans enchantement, sans rêves. Il s'agit de la capacité de révéler un monde de possibles en captant les forces et les potentialités d'un sujet et en le traduisant avec lui dans un projet stimulant.

L'objet de ce livre réside dans la conceptualisation d'un processus, celui de la relation thérapeutique qu'un éducateur, dans un vécu partagé, propose à un sujet, particulièrement dans le cadre d'une rencontre hebdomadaire que j'appelle la rencontre d'accompagnement clinique. Elle consiste en une écoute des désirs intrapsychiques, une analyse des phénomènes transférentiels et contretransférentiels, où l'éducateur et le jeune travaillent sur le sens du vécu.

Ce type de rencontre s'inscrit dans la tradition psychoéducative. Le modèle psychoéducatif se définit à l'aide de trois dimensions : l'organisation et l'animation d'expériences dans un programme structuré et/ou dans des activités, de même que l'utilisation de ces expériences vécues. La dimension clinique de l'intervention psychoéducative réside dans l'utilisation de ces expériences vécues. Cette opération professionnelle importante, l'utilisation, servira de clé de voûte à la rencontre d'accompagnement clinique.

Le concept d'utilisation, se définit comme étant «la capacité de déclencher la prise de conscience du sujet et de le mobiliser à adapter des moyens dans la perspective d'une évolution vers une plus grande adaptation. [...] l'utilisation doit permettre au sujet de faire des liens entre ses attitudes, ses comportements et ce qui se passe dans son monde intérieur. » (David et Tanguay, 1998) C'est un processus dynamique, l'établissement de liens entre des catégories se présentant dans les psychopathologies et l'inadaptation comme dénouées, voire morcelées.

L'éducateur, par son intervention, travaille à faire basculer le regard du sujet de la scène externe à la scène interne, vers son monde intérieur. Il cherche à déclencher une prise de conscience, créer une sorte de court-circuit dans le système de défense, créer un déséquilibre, voire mieux, une question. 
Pour bien utiliser les événements, l'éducateur, doit posséder une conception du fonctionnement psychique afin de comprendre le sens des comportements qu'il observe dans son travail clinique. J'ai choisi de décrire un modèle qui laisse une place à la capacité de rêver de l'éducateur.

L'un des principaux ouvrages de Bion, Aux sources de l'expérience (1979), fournit un modèle de référence théorique sur la notion d'expérience et de sa place dans l'appareil psychique, en plus de donner des pistes de réflexion sur l'intervention.

Bion (1979, 38), évoquant des sujets présentant des difficultés de pensée importantes, à l'instar de la majorité de la clientèle des Centres Jeunesse, parlera de leurs attaques contre l'activité de liaison. Ces personnes, par exemple, détruisent les liens psychiques que nous tentons d'établir avec elles en traitement. Elles détruisent les relations interpersonnelles. Le mécanisme de brisure relationnelle, si bien décrit par Michel Lemay (1979), en est une manifestation mortifère.

On peut se demander ce qui caractérise ces pensées qu'il faut détruire pour survivre. Si la clientèle des Centres Jeunesse, pour une large part, se compose de sujets présentant des troubles de la pensée, nous pourrions nous questionner sur la nature des expériences que ces jeunes ont vécues. Bion décrit une pensée très archaïque, c'est-à-dire dès les premiers temps de l'activité psychique, ces expériences ont donc été vécues très tôt dans la vie. Les enfants victimes de négligence m'apparaissent être particulièrement vulnérables à ce type de difficultés. En effet, ces enfants, dans leur solitude, ressentent «des sentiments de dépression, de persécution, de culpabilité, des éléments hallucinatoires liés par le sentiment d'une catastrophe, d'une mort psychique. Ce sont des impressions sensorielles, des vivances émotionnelles dont le nourrisson doit se libérer en les expulsant par identification projective ${ }^{5}$ ». Ce mécanisme de défense évacue les éléments qui ne peuvent être psychisés parce qu'indicibles, non représentés. L'expérience, ici une terreur sans nom, ne peut être intégrée à l'appareil psychique. Les éléments qui intoxiquent la psyché, s'ils y restent, détruisent tout: «[...] le sujet évacue dans l'autre les éléments $[\ldots]$ qu'il ne peut contenir (le terme est important) dans sa psyché. Ce sera à l'autre de contenir ${ }^{6}$.»

Cette fonction de contenant ayant été défaillante chez les parents de plusieurs enfants placés en centre de réadaptation, ces jeunes sont souvent incapables de fonctionner dans des endroits de vie où le contenant n'est pas suffisamment solide pour eux. Ils ont besoin d'une structure externe, leur monde interne n'étant pas suffisamment construit, afin de les contenir et éviter qu'ils ne se détruisent ou détruisent l'autre. Ces jeunes sont de plus caractérisés par une incapacité à apprendre de leur expérience. Ils ne tirent pas profit de l'expérience. Cela s'explique, selon Bion, par le fait que ces jeunes souffrent des troubles de la pensée : «défaut d'élaboration psychique, absence de liaisons ou destructions des liens déjà établis avec la réalité, pauvreté du matériel onirique et fantasmatique, etc » $(1979,4)$.

Revenons sur la notion de contenant, car elle s'avère une idée particulièrement intéressante pour le travail avec les personnes souffrant de troubles de la pensée et qui constituent une partie importante de la clientèle des Centres Jeunesse. 
À l'instar d'une mère, lorsque l'enfant utilisera l'identification projective, l'institution devient le contenant, l'équipe et chacun de ses membres contiendront ses sensations, affects, pulsions, idées, les penser, afin de les lui redonner.

«Devant une expérience de faim, véritable fin du monde, la mère va venir tempérer cette explosion par ses paroles. « $\mathrm{Oh}$, tu as faim, tu veux manger». Elle donne du sens à ses éprouvés corporels, elle lui permet de les psychiser. Mais si, à la place de ces paroles, elle lui change sa couche, l'enfant aura du mal à psychiser cet éprouvé de faim.

Cette expérience du bébé est transposable au vécu adulte. L'autre est indispensable dans la symbolisation, dans la création de l'appareil psychique. L'autre est indispensable pour contenir, pour penser ce qui n'est pas pensable. On peut ainsi entrevoir la place de l'autre dans ce processus thérapeutique ${ }^{7}$.»

On le voit la théorie de Bion offre aux intervenants qui travaillent au Centre jeunesse une armature conceptuelle qui permet de penser la clinique d'une façon intéressante.

Afin de préparer les rencontres d'accompagnement clinique, j'ai adapté et développé un outil, La Grille de retour sur le vécu, du psychanalyste Allaire. Encore ici, un outil pour penser, convivial et efficace qui permet de formuler des hypothèses sur la représentation du monde intérieur d'un sujet et sur ce qu'il tente de dire à son entourage. Je l'ai adapté en me centrant sur les catégories : agi, senti et dit.

La rencontre d'accompagnement devient alors le lieu privilégié pour aviver les activités de liaison entre les trois catégories. J'ai conceptualisé trois activités que l'éducateur doit effectuer. Une première consiste en une mise en forme du matériel d'observation; La Grille de retour sur le vécu servira d'outil. Ensuite, vient l'activité de mise en sens (la fonction contenant et l'activité de rêve de Bion); et finalement, l'activité de mise en mots.

Enfin, il est important de mentionner l'utilité essentielle, lors de la rencontre d'accompagnement, des notions de projet et d'espoir qui permettent l'articulation concrète d'un travail clinique de mise en forme, de mise en sens et de mise en mots.

\section{Conclusion}

La pratique clinique en Centre de réadaptation propose des défis cliniques passionnants. Elle constitue un travail difficile auprès d'une clientèle complexe et exige la compréhension des problématiques par l'analyse des dynamiques individuelle, familiale et institutionnelle.

Dans les années qui viennent, je serai particulièrement intéressé à travailler sur la question des jeunes qui arrivent avec des besoins de réadaptation très importants et avec, en plus, un diagnostic psychiatrique. Le défi sera de développer davantage 
un travail interdisciplinaire complémentaire afin de mieux comprendre l'imbrication des diverses pathologies les une sur les autres et aussi d'articuler un traitement non plus seulement en parallèle, mais dans une perspective de systèmes complexes.

\author{
daniel puskas \\ 9437 lajeunesse \\ montréal, québech2m 1s5 \\ dpuskas@sympatico.ca
}

\title{
Notes
}

1. D'abord établissons notre terminologie. J'aimerais faire tout de suite la distinction entre deux concepts retrouvés dans la littérature, soit celui de l'intergénérationnel et celui du transgénérationnel. L'intergénérationnel est ce qui se passe entre les générations, comme en fait foi le préfixe inter, tandis que le transgénérationnel est ce qui traverse les générations, comme le suggère le trans. Dans ces deux cas ces termes sont employés généralement comme adjectifs. Dans l'ensemble de mes lectures je n'ai pas retrouvé de terme spécifique nommant les phénomènes transgénérationnels, je propose donc d'utiliser ces termes, celui d'intergénérationnel et de transgénérationnel également comme substantif. Qu'il me soit permis tout de suite de prendre parti entre ces deux concepts. Pour moi l'intergénérationnel est une des manifestations du transgénérationnel. Il faut donc analyser ce qui se passe entre les générations pour comprendre ce qui les traversent. En ce sens le trans subsume l'inter, celuici étant un cas particulier de celui-là. Afin d'éviter des lourdeurs dans le texte je me référerai à présent au seul concept de transgénérationnel, sauf avis contraire, auquel cas je spécifierai.

2. In : «La pensée complexe».

3. Le terme intervenant est ici pris dans sa large acceptation et inclut toute personne qui intervient en relation d'aide.

4. L'essentiel des informations a été recueillies sur les sites suivants:

http://www.chez.com/siubhan/tissage/attrape.htm

http://www.capteurdereves.org/

http://www.ciac.ca/biennale/fr_expositions.html

http://angelsplace.club.fr/Chamanisme.htm

http://www.artindien.com/squaw.html

5. Bion, http://psychiatriinfirmiere.free.fr/infirmiere/formation/psychiatrie/enfant/therapie/bion.htm

6. http://philautarchie.net/viewtopic.php?t=15

7. Bion, http://philautarchie.net/viewtopic.php?t=15

\section{Bibliographie}

Bion, W.R., 1979, Aux sources de l'expérience, Paris, PUF.

David L., Tanguay Y, 1998, Cahier de stage Pse 3117, Université de Montréal.

Guérin P.J., Eileen G., Pendagast E.G., 1976, Evaluation of Family Systems and Genogram, in: Guérin, Family Therapy Theory and Practice, Gardner Press, N.Y., 450-467.

Lemay M.,1979, J'ai mal à ma mère, Fleurus, Paris.

McGoldwich, M., Gerson, R., 1990, Génogramme et entretien familial, E.S.F., Paris.

Puskas D., 2002, Amours clouées, Éd. Sciences et culture. P.23. 